\title{
Thiazolidinediones versus metformin on improving abnormal liver enzymes in patients with type 2 diabetes mellitus: a meta- analysis
}

\author{
Chunmei Xu ${ }^{1, *}$, Junyu Zhao ${ }^{1, *}$, Xiaojun Zhou ${ }^{1}$, Rui Zhang ${ }^{1}$, Tianyue Xie ${ }^{3}$, Zhiwei \\ Zou $^{2}$, Lin Liao ${ }^{1}$ and Jianjun Dong ${ }^{2}$ \\ ${ }^{1}$ Department of Endocrinology, Shandong Provincial Qianfoshan Hospital, Shandong University, Jinan, China \\ ${ }^{2}$ Department of Medicine, Division of Endocrinology, Qilu Hospital of Shandong University, Shandong University, Jinan, China \\ ${ }^{3}$ Department of Endocrinology, Shandong Provincial Qianfoshan Hospital, Shandong University of Traditional Chinese \\ Medicine, Jinan, China \\ "Co-first authors and equal contribution to this work
}

Correspondence to: Lin Liao, email: liaolin009@sina.com

Jianjun Dong, email: cwc_ll@sdu.edu.cn

Keywords: liver enzyme abnormalities; thiazolidinediones; metformin; randomized controlled trials; meta-analysis

Received: August 01, $2017 \quad$ Accepted: December 05, $2017 \quad$ Published: January 13, 2018

Copyright: $\mathrm{Xu}$ et al. This is an open-access article distributed under the terms of the Creative Commons Attribution License 3.0 (CC BY 3.0), which permits unrestricted use, distribution, and reproduction in any medium, provided the original author and source are credited.

\section{ABSTRACT}

Background: Liver enzyme abnormalities are common in patients with type 2 diabetes. Currently, the inverse relationship between elevated liver enzymes and antidiabetics intake may be explained by rigorous treatment and good control. However, few studies have directly explored the influence of antidiabetics on abnormal liver function, especially the comparison between two insulin sensitizersthiazolidinediones and metformin.

Materials And Methods: Databases, including PubMed, Cochrane, CNKI, Wanfang and VIP were searched. Two reviewers performed independently. Meta-analysis was used when studies were homogeneous enough.

Results: Six studies, including $\mathbf{4 7 2 6}$ patients with type 2 diabetes, were involved in this systematic review. Compared with metformin, thiazolidinediones significantly reduced the alanine transaminase, aspartate aminotransferase and gamma-glutamyl transpeptidase. Further subgroup analysis suggested that pioglitazone-treated participants showed vast improvement in decreasing alanine transaminase (MD = $-13.70 ; 95 \% \mathrm{CI}=-16.91$ to $-10.52 ; P<0.00001 ; I^{2}=1 \%$ ), aspartate aminotransferase $\left(M D=-3.51 ; 95 \% C I=-5.74\right.$ to $\left.-1.28 ; P=0.002 ; I^{2}=0 \%\right)$ and gamma-glutamyl transpeptidase $\left(M D=-5.41 ; 95 \% C I=-9.40\right.$ to $-1.42 ; P=0.008 ; I^{2}=0 \%$ ), while rosiglitazone exhibited no difference in lowering corresponding liver enzyme levels. Besides, thiazolidinediones similarly decreased fasting plasma glucose. However, thiazolidinediones were inferior to metformin in lowering HbA1C and alkaline phosphatase. Additionally, no significant publication bias was seen.

Conclusions: Thiazolidinediones may confer modest biological improvement of liver function in people with type 2 diabetes than metformin. But owing to the limited methodological quality, more clinical researches are warranted in the future.

\section{INTRODUCTION}

Diabetes mellitus (DM), which is a common chronic disease giving rise to numerous complications, has aroused the public's attention around the world. Currently, metformin has become the pharmacological cornerstone in the treatment for patients with type 2 diabetes mellitus (T2DM) [1]. When metformin does not suffice or is contra-indicated, the alternative oral treatment options are: sulphonylureas (SUs), a-glucosidase inhibitor, thiazolidinediones (TZDs), dipeptidyl peptidase-4 (DPP-4) inhibitors [2]. It has been shown that the prevalence of elevations of alanine 
transaminase (ALT) was 3 to 4 times higher in patients with T2DM than in patients without diabetes [3]. Furthermore, patients with DM and fatty liver are remarkably insensitive to insulin. Most of patients with fatty liver or non-alcoholic steatohepatitis (NASH) typically have mildly elevated aminotransferase enzyme levels which frequently oscillate in and out of the normal range. Thus for patients with T2DM, it is significant to perform pre-treatment liver tests in order to distinguish the reasons for later liver enzyme abnormalities as drug-induced liver damage or not [4]. Yet, currently there are limited established studies monitoring liver function among patients with T2DM applying oral antidiabetics and exploring the specific effects of these hypoglycemic agents on improving liver function. In addition, NASH is the main hepatic pathologic manifestation that concomitantly occurs to diabetics. Nowadays, no effective measures could cure NASH other than lifestyle modification and weight loss, which are often of trouble to achieve and even harder to maintain [5-7]. TZDs and metformin, as the two main groups of insulin-sensitizing drugs, may offer the therapeutic benefits for NASH treatment [8]. Despite of the widespread use of insulin-sensitizing drugs in clinical practice, a growing number of patients began to be concerned about the side effects of insulin sensitizers, including abnormality of renal function, and in particularly, liver damage. The purpose of this meta-analysis of randomized controlled trials is to summarize the currently available evidence for the effect of insulin-sensitizing agents on biochemical endpoints regarding both liver function and plasma glucose level in diabetics.

\section{MATERIALS AND METHODS}

\section{Searching methods for identification of studies}

A systematic search was undertaken by two independent reviewers through searching the following database: PubMed, Cochrane, CNKI, VIP and Wanfang data with disagreements resolved by consensus. There were various searching words for a variety of databases. The searching terms we used were related to interventions, disease and outcome indicators. Our searching strategy included as following: (Thiazolidinediones OR Thiazolidinedione OR TZDs OR rosiglitazone OR pioglitazone) AND (metformin OR glucophage) AND (Liver function OR Alanine Transaminase OR ALT OR Aspartate Aminotransferases OR AST OR gammaGlutamyltransferase OR GGT OR Alkaline Phosphatase OR AKP) AND (RCT OR controlled clinical trial OR randomized). Databases were searched from the earliest data to 1 July 2017.

\section{Inclusion and excluded criteria}

Two reviewers independently browsed through the titles and the abstracts identified according to the above- described strategy. And they reached consensus through consultation. If an agreement couldn't be reached, a third reviewer would decide. All potentially relevant essays were retrieved, and the full text of these studies was read over to determine which trials satisfied the inclusion criteria. Inclusion criteria were randomized controlled clinical trials of patients with T2DM with abnormal liver function tests, who were treated with thiazolidinedione drugs whether with rosiglitazone or pioglitazone of any dose versus metformin of any dose, with or without other interventions or healthy lifestyle management such as diet control or exercise. Conversely, Non-RCT trials or/ and trials of patients with non-T2DM or/and trials without TZDs and metformin for comparison or/and liver enzymes not mentioned were excluded.

\section{Data extraction}

The two authors extracted the following data for all included trials independently. Items included were title, author, country, date, number of participants, inclusion and exclusion criteria, mean (or median) age, sex ratio, intervention and dose, follow-up length, duration of diabetes and/or of additional intervention(s), and outcome measures. The characteristics of the articles were recorded in a form.

\section{Methodological quality and risk of bias}

We assessed the influence of methodological quality to avoid the risk of overestimation of intervention effects. The specific assessment methods were used via Cochrane definitions: generation of the allocation sequence (high or low or unclear risk); allocation concealment (high or low or unclear risk); blinding of participants and personnel (high or low or unclear risk); blinding of outcome assessment (high or low or unclear risk); incomplete outcome data (high or low or unclear risk); selective reporting (high or low or unclear risk). Two reviewers independently evaluated these items.

\section{Missing data}

Where data were missing, corresponding data were extracted from other sources. On condition that data had not been reported sufficiently or were not published at all, we would correspond with the authors to acquired further information.

\section{Data analysis}

The main outcomes were the change of biochemical parameters from baseline, including serum activities of alanine transaminase (ALT), aspartate aminotransferase (AST), gamma-glutamyl transpeptidase (GGT), alkaline phosphatase (AKP). Besides the efficacy of the 
hypoglycemic drugs on the liver, change of fasting plasma glucose (FPG) and glycosylated hemoglobin (HbA1C) [9] were also examined. The statistical package that RevMan5.3 and Stata version 12.0 (StataCorp, College Station, TX, USA). provided was used. For continuous variables, the mean difference (MD) with $95 \%$ interval was calculated. The fixed-effects model was applied to synthesize the data of the different trials when there were no significant heterogeneities; otherwise, the randomeffects model was used [10]. Subgroup analysis by kind of TZDs (either rosiglitazone or pioglitazone) was applied to assess the efficacy of different therapeutic group. The $\mathrm{I}^{2}$ was calculated which can be interpreted as the percentage of the variation between studies that attributes to heterogeneity rather than chance. $\mathrm{I}^{2}=0 \%$ indicates no heterogeneity, and $\mathrm{I}^{2}=100 \%$ represents that all variation derives from heterogeneity. Sensitivity analyses excluding one study at a time were also performed to evaluate whether any specific study significantly influenced the overall pooled results. Publication bias was evaluated via Begg's funnel plots [11]. Statistical levels of significance were estimated with $P<0.05$.

\section{RESULTS}

\section{Search results}

Our initial search identified 294 references from PubMed and Cochrane, and no reference gained from Chinese database according to the agreement for study selection. After getting rid of the duplicate, 282 references remained to be looked through the abstracts. Only 25 references were assessed for full-text review because of the failure of study design or intervention or disease or outcome measure. We excluded 19 references for the previous reasons, the remaining six references were considered for inclusion. And the searching progress was displayed in Figure 1.

\section{Study characteristics}

Among the final six references, two studies were conducted in Europe [12-13], one in Greek [14], one in Japan [15], one in Turkey [16], and one in Europe, Canada, and Australia [17]. The main characteristics of the studies were shown in Supplementary Table 1. The six randomized controlled trials [12-17] were published as full text articles and included 4726 patients. The sample size of each arm varied apparently, ranging from 14 to 1857 participants. The experimental intervention was TZDs, and we chose rosiglitazone $[14,16]$ and pioglitazone $[12,13,15,17]$ as intervention measures, except for troglitazone due to the known hepatotoxicity $[18,19]$. The trials we included contained not only metformin or TZDs monotherapy but also adding-on to SUs $[12,13]$. Only in the T.Karo 2009 et al. trial, 50 patients received one-month exercise therapy including $50 \mathrm{~min}$ or more of exercise per week [15]. All except for two trials $[12,13]$ of the group, patients received diet. In the F.Iliadis 2007 et al. trial [14], all participants suffered from T2DM concomitantly with NASH. The durations of the intervention periods varied from 12 to 52 weeks.

\section{Methodological Quality}

Of all studies, four studies mentioned the specific randomized method [12, 14-16], but the remaining two referred to "random" but no method in detail $[13,17]$. Two studies adopted allocation concealment [15, 17], and five studies were double-blind [12-15, 17]. Apart from the T.Karo 2009 et al. trial [15], other five studies had incomplete outcome data bias. And the reporting bias was only mentioned in the G.Belcher 2004 et al. trial [13]. In brief, the methodological quality of the included studies we had them in this meta-analysis was not good.

\section{Outcomes}

\section{Liver function tests}

\section{ALT with monotherapy}

All of the six studies [12-17] were available to investigate the serum activities of ALT, and the total number of participants was 2832 and 1894 respectively for TZDs or metformin monotherapy. The pooled results of six RCTs revealed a significant difference with high heterogeneity on the change of serum ALT levels from baseline in patients treated with TZDs compared with metformin. Considering that different class of TZDs was applied by different studies, subgroup analysis of the fixed-effects model was conducted. Rosiglitazone group [14-16] displayed the moderate heterogeneity but no difference of the reduction of ALT (MD $=0.67 ; 95 \% \mathrm{CI}$ $=-4.58$ to $\left.3.24 ; P=0.14 ; \mathrm{I}^{2}=53 \%\right)$. Due to substantial heterogeneity in the group treated with pioglitazone [12, $13,15,17]$, we conducted sensitivity analysis by excluding a study conducted by M.Hanefeld et al. [12] for addingon to SUs as therapy. The result found a more significant decreased effect on ALT by pioglitazone than metformin $\left(\mathrm{MD}=-13.72 ; 95 \% \mathrm{CI}=-16.91\right.$ to $-10.52 ; P<0.00001 ; \mathrm{I}^{2}$ $=1 \%$ ) (Figure $2 \mathrm{~A})$.

\section{AST with monotherapy}

Another evaluating indicator of liver enzymes, AST, was assessed from included five trials [12, 13, 15-17]. Similarly, subgroup analysis of the fixed-effects model was conducted by grouping for the class of TZDs, and the result showed that there was no difference in the reduction of AST between the rosiglitazone group and metformin group with unmeasurable heterogeneity for only one study included [16] (MD $=-1.10 ; 95 \%$ CI 
$=-7.66$ to $5.46 ; P=0.74)$. However, pioglitazone was more effective than metformin in the reduction of AST $\left(\mathrm{MD}=-3.51 ; 95 \% \mathrm{CI}=-5.74\right.$ to $-1.28 ; P=0.002 ; \mathrm{I}^{2}=$ $0 \%$ ) with no heterogeneity after excluding one trial that was responsible for heterogeneity for its adding-on to SUs intervention [12] (Figure 2B).

\section{GGT and AKP with monotherapy}

Four studies [12-14, 17] measured the biochemical response of GGT and AKP. And the fixed-effects model was used to merge $\mathrm{MD}$ values and the pooled MD was $-3.76(95 \% \mathrm{CI}:-7.48$ to $-0.04 ; P=0.05)$ with moderate heterogeneity $\left(P=0.08 ; \mathrm{I}^{2}=55 \%\right)$ for GGT, which indicated that TZDs would decrease GGT more remarkablely than metformin. Further subgroup analysis result indicated that rosiglitazone had similar efficacy in reducing GGT level with metformin $(\mathrm{MD}=7.00 ; 95 \% \mathrm{CI}$ $=-3.20$ to $17.20 ; P=0.18)$. By contrast, pioglitazone significantly decreased GGT compared with metformin $\left(\mathrm{MD}=-5.41 ; 95 \% \mathrm{CI}=-9.40\right.$ to $\left.-1.42 ; P=0.008 ; \mathrm{I}^{2}=0 \%\right)$ (Figure 2C). Due to the substantial heterogeneity from these studies $\left(P=0.0005 ; \mathrm{I}^{2}=83 \%\right)$, subgroup analysis of the fixed-effects model was used to merge MD values of AKP. The meta-analysis found that there was statistically significant reduction between participants treated with rosiglitazone and metformin $(\mathrm{MD}=-14.00 ; 95 \% \mathrm{CI}=$ -22.75 to $-5.25 ; P=0.002$ ) with undetected heterogeneity for only including one study. Besides, the pooled data for pioglitazone group was $2.64(95 \% \mathrm{CI}=1.51$ to $3.77 ; P$ $<0.00001)$ with moderate heterogeneity $\left(P=0.13 ; \mathrm{I}^{2}=\right.$ $51 \%$ ), which indicated that pioglitazone was inferior to metformin in lowering AKP (Figure 2D).

\section{Adding-on to SUs}

Apart from monotherapy, treatment with adding-on to SUs was reported by two studies. One research which was excluded by above meta-analysis was conducted by M.Hanefeld et al. [12]. and only used combination therapy with adding-on to SUs as intervention. Another study performed by G.Belcher et al. [13]. applied with both monotherapy and combination treatment with SUs. Among the two studies adding-on to SUs, the decrease of ALT from baseline to the end of treatment was more significant when treated with TZDs in comparison with metformin with a MD of $-2.34(95 \% \mathrm{CI}:-3.80$ to -0.89 ; $\left.P=0.002 ; \mathrm{I}^{2}=76 \%\right)$. As to the reduction of AST, the result showed that TZDs was inferior to the treatment of metformin with adding-on to SUs, and the pooled data was a MD of 1.12 (95\% CI: 0.08 to $2.15 ; P=0.04 ; \mathrm{I}^{2}=16 \%$ ). However, there was no significant difference in GGT reduction with pioglitazone compared with metformin, with a MD of $-3.00\left(95 \% \mathrm{CI}:-7.71\right.$ to $1.71 ; P=0.21$; $\mathrm{I}^{2}$ $=0 \%$ ). Similar to the change of AST, analysis indicated that metformin with adding-on to SUs showed a more significant role in the reduction of AKP compared to TZDs $\left(\mathrm{MD}=5.00 ; 95 \% \mathrm{CI}: 3.16\right.$ to $\left.6.84 ; P<0.00001 ; \mathrm{I}^{2}=0 \%\right)$ (Figure 3).

\section{Efficacy on lowering glucose}

\section{FPG}

Four studies [12, 14-16] contributed to the FPG analysis with a total of 758 participants, 378 and 380 in the TZDs and metformin group, respectively. There was no difference of the change of FPG from baseline to the end of treatment in four trials by calculating the $\mathrm{MD}(\mathrm{MD}=0.05 ; 95 \% \mathrm{CI}:-0.22$ to 0.32$)$. However, this result was built around the premise that heterogeneity was higher we couldn't neglect $\left(P=0.009 ; \mathrm{I}^{2}=74 \%\right)$. Hence, to eliminate the heterogeneity, the subgroup analysis was performed. For one group treated with rosiglitazone [14, $16]$, the heterogeneity still existed $\left(P=0.006 ; \mathrm{I}^{2}=87 \%\right)$ and the pooled MD was $-0.38(95 \% \mathrm{CI}:-0.89$ to $0.13 ; P=$ $0.15)$, which showed no difference between rosiglitazone and metformin. And for another group with pioglitazone $[12-15,17]$, the fixed-effects model was used to merge MD values of FPG and a similar result was found with the pooled MD was 0.21 (95\% CI: -0.10 to $0.53 ; P=0.18)$ with no heterogeneity $\left(P=0.54 ; \mathrm{I}^{2}=0 \%\right)$ (Figure $\left.4 \mathrm{~A}\right)$.

\section{HbA1C}

The same four studies [12, 14-16] as reporting FPG were available to measure the level of $\mathrm{HbA1C}$. Compared to the participants with TZDs, metformin was more effective with the pooled MD of 0.14 (95\% CI: 0.02 to 0.27$)$ with moderate heterogeneity $\left(P=0.15 ; \mathrm{I}^{2}=\right.$ $43 \%$ ). Nevertheless, due to the reduction by 0.27 at most of $\mathrm{HbA} 1 \mathrm{C}$ in the metformin group than TZDs group, the effective of metformin on $\mathrm{HbA} 1 \mathrm{C}$ reduction might have little clinical significance (Figure 4B).

\section{Publication bias}

The funnel plots based on the ALT (Begg's test: $P$ $=0.707$ ) and AST (Begg's test: $P=0.806$ ) are shown in Figures $5 \mathrm{~A}$ and $5 \mathrm{~B}$, respectively. The funnel plot shapes were symmetrical, indicating no obvious reporting bias.

\section{DISCUSSION}

Liver enzyme abnormalities are common in patients with T2DM [20]. It has been shown that the prevalence of elevations of ALT was 3-4 times higher in patients with T2DM than in patients without T2DM [3]. Thus, for insulin-resistant T2DM, it is crucial to perform pretreatment liver tests in order to be able to interpret later liver enzyme abnormalities observed after the initiation of drug treatment [4]. Insulin resistance seems to play 
a central role of accumulation of triglycerides in the liver and initiation of the inflammatory cascade. That is why insulin sensitizers can be regarded as beneficial treatment at this stage of liver injury [21]. As the top two representative drugs of insulin-sensitizers, TZDs and metformin are widely applied in clinics for diabetes [22-24]. It is worth noting that the first-generation TZD, troglitazone, the first compound approved by the Food and Drug Administration in the US, proved to be hepatotoxic and was withdrawn from the market after the report of several dozen deaths or cases of severe hepatic failure requiring liver transplantation [4]. However, the second-generation thioglitazones, that is, rosiglitazone and pioglitazone appear to be safer, although their use is currently contraindicated in the presence of active liver disease or of ALT more than 2.5 times normal [25]. Nowadays, there is no published study comparing the efficacy of the two insulin-sensitizers on the liver function.

Earlier systematic reviews have shown improvements across the histological and biological response of the liver treated with hypoglycemic drugs, while these researches studied people who suffered from NASH or NAFLD, not with T2DM. A systematic review performed by Suzanne E. et al. indicated that TZD treatment may be less effective in non-diabetics with NASH [26]. Nevertheless, an open-label, randomized, a single-center study revealed that rosiglitazone therapy seemed to be more effective in metabolic control and histological improvement in NAFLD patients with impaired glucose metabolism and metformin therapy alone has not improve serum transaminase levels [27]. In addition, another clinical trial showed that insulin sensitizers could lead to an improvement in metabolic, biochemical and histological parameters in NASH as a result of improved insulin sensitivity [28].

Since increased transaminase activity is an indicator of hepatic necroinflammation which could lead to fibrosis and cirrhosis of liver [29], transaminase level was regarded as an important outcome for monitoring liver function. Our results indicated that the level of ALT, a useful marker for measuring hepatocellular damage and reflecting cell membrane function with the highest concentration found in liver [30], was significantly reduced for the patients treated with TZDs than that of metformin group, especially treated with pioglitazone. Similar conclusions that long-term therapy with pioglitazone may be necessary to maintain improvements in serum aminotransferase in patients with $\mathrm{NASH}$, while improvements in serum aminotransferase levels treated with metformin may not be sustained for even 48 weeks were reached in several studies $[22,25,31]$. Similar with ALT, there was statistically significant difference in the change of AST between treatment and control group. ALT and AST levels, measure the concentration of intracellular hepatic enzymes that have leaked into the circulation and serve as a marker of hepatocyte injury [32]. Some potential explanations for elevated transaminases in insulin-resistant states include oxidant stress from reactive lipid peroxidation

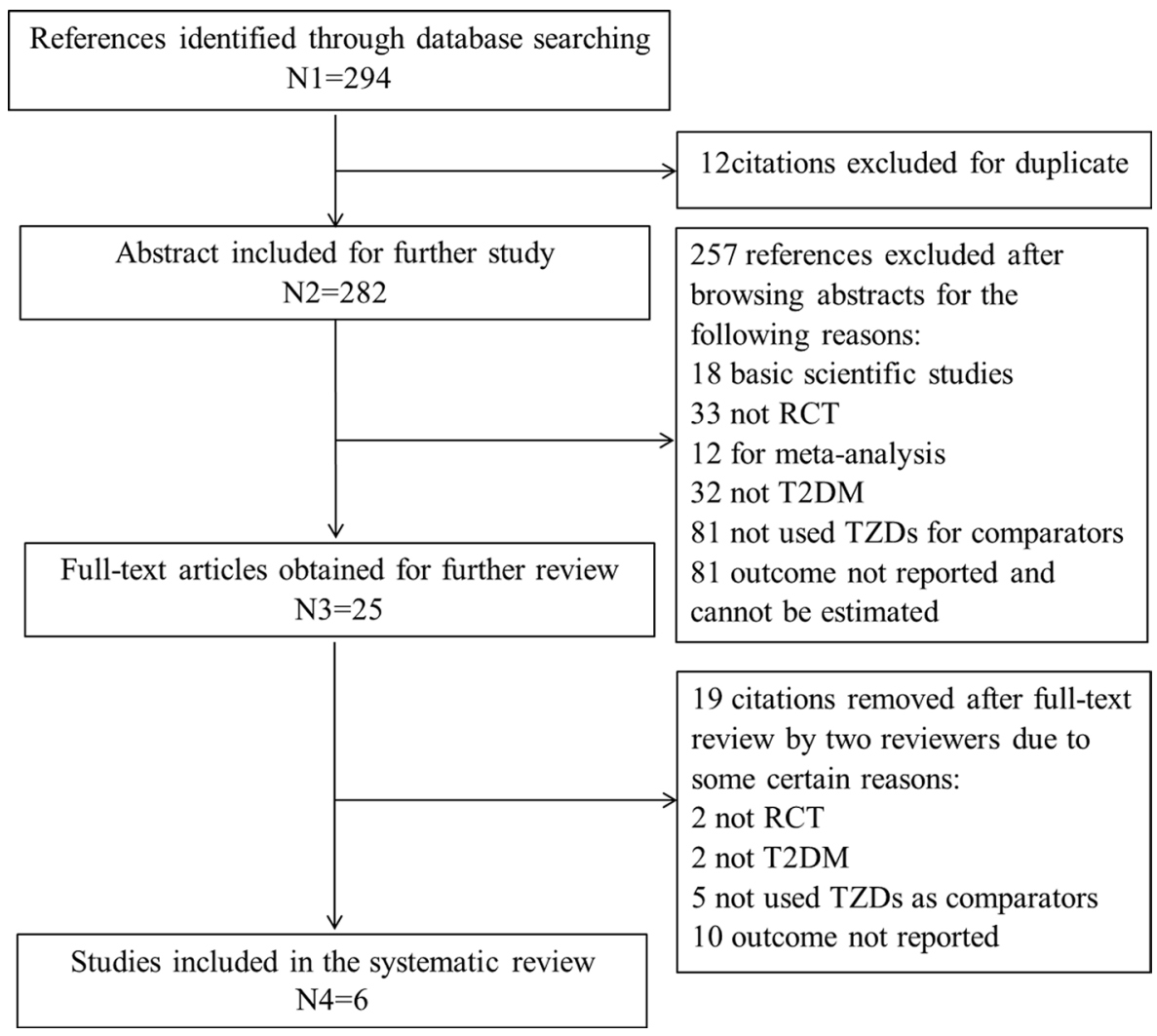

Figure 1: Literature search flow diagram. 
and recruited inflammatory cells. And the insulinresistant state is also characterized by an increase in proinflammatory cytokines such as tumor necrosis factor- $\alpha$ (TNF- $\alpha$ ), which may also contribute to hepatocellular injury [32]. Indeed, a few possible mechanisms have been explored to explain the hepato-protective effects of TZDs, including amelioration of insulin resistance, reducing the TNF- $\alpha$ production, increasing adiponectin concentration, activation of AMP-related protein kinase and inactivation of the intracellular pro-inflammatory signaling pathway [33-36]. Besides, the ability to improve liver enzymes of TZDs may be also explained by activation of PPAR- $\gamma$, then causing downregulation of inflammation and fibrosis through its effect on Kupffer and hepatic stellate cells [27, 37].

As for the other markers for liver function tests, applying TZDs would decrease GGT more remarkablely, while metformin showed stronger efficacy in lowering

A

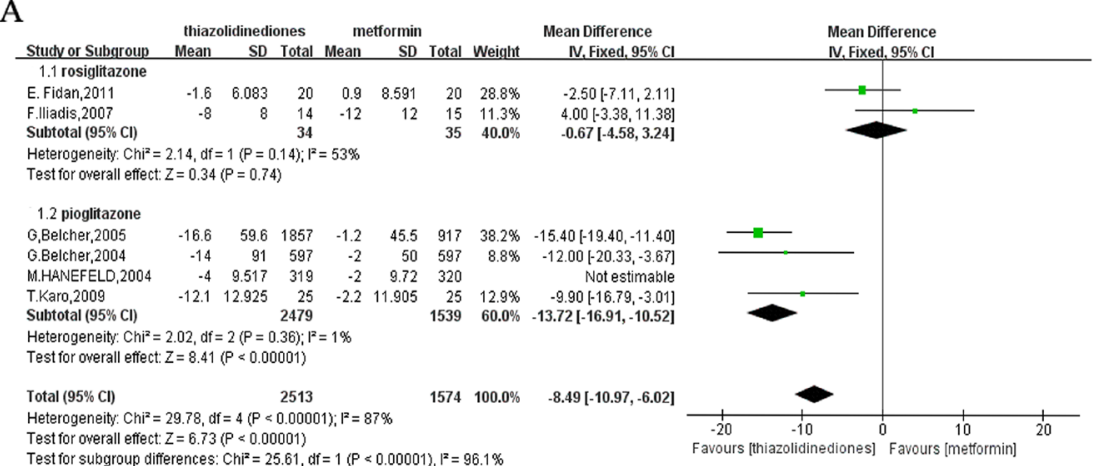

Test for overall effect: $Z=6.73(P<0.00001)$
Test for subgroup differences: $C \mathrm{I}^{2}=25.61$, df $=1(P<0.00001), P^{2}=96.1 \%$

B

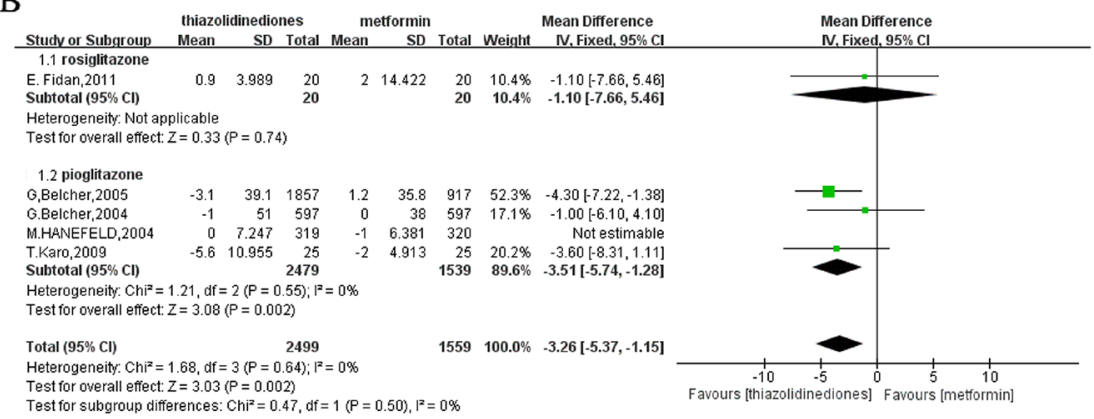

Test for overall effect: $Z=3.03(P=0.002)$
Test

Test for subgroup differences: $\mathrm{Chi}^{2}=0.47, \mathrm{df}=1(P=0.50), F^{2}=0$ S

C

thiazolidinediones metformin Mean Difference Mean Difference

Studv or Subgroup Mean SD Total Mean SD Total Weight N, Fixed, 95\% Cl N. Fixed, 95\%

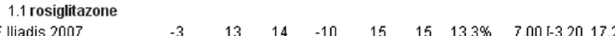

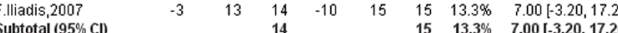

Heterogeneity. Not applicable

Test for overall effect: $Z=1.35(P=0.18$

1.2 pioglitazone

$\begin{array}{lllllllll}\text { G,Belcher,2005 } & -17.6 & 112.1 & 1857 & -8.7 & 62.8 & 917 & 32.5 \% & -8.90[-15.42,-2.39\end{array}$

$\begin{array}{llllllllll}\text { G.Belcher, } 2004 & -12 & 84 & 597 & -8 & 74 & 597 & 17.1 \% & -4.00[-12.98,4.98]\end{array}$

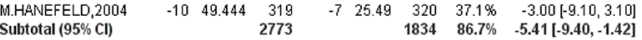

2773
Heterogeneity $\mathrm{Chi}^{2}=1,79, \mathrm{df}=2(\mathrm{P}=0.41): \mathrm{F}^{2}=0$

Heterogeneity: $\mathrm{Chi}^{2}=1.79, \mathrm{df}=2(P=0.41)$,
Test for overall effect: $Z=2.66(P=0.008)$

Total $(95 \% \mathrm{Cl})$

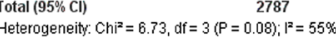

Test for overall effect $Z=1$, 98 P $P=0.00$,

Test for subgroup differences: Chi $^{2}=4.93$, df $=1(P=0.03),\left.\right|^{2}=79.70$

$1849 \quad 100.0 \% \quad-3.76[-7.48,-0.04]$

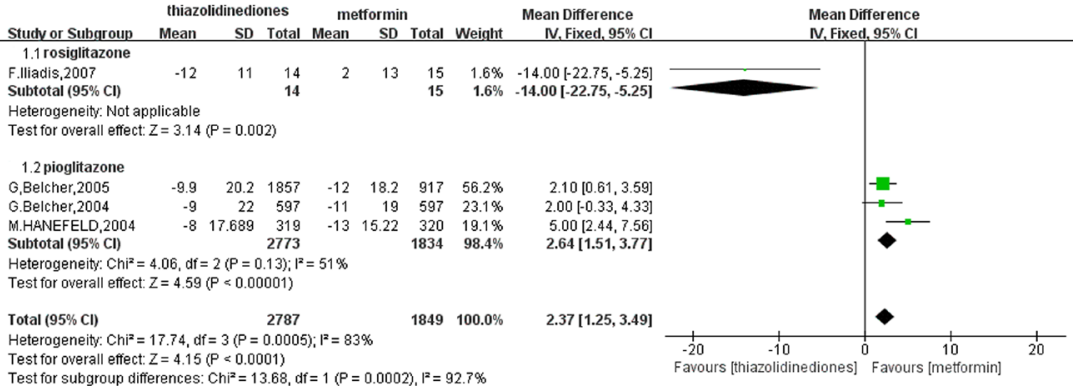

Figure 2: Forest plot of the improvement of liver enzymes from baseline to the end of treatment with monotherapy. (A) ALT. (B) AST. (C) GGT. (D) AKP. ALT: alanine transaminase; AST: aspartate aminotransferase; GGT: gamma-glutamyl transpeptidase; AKP: alkaline phosphatase. 
A

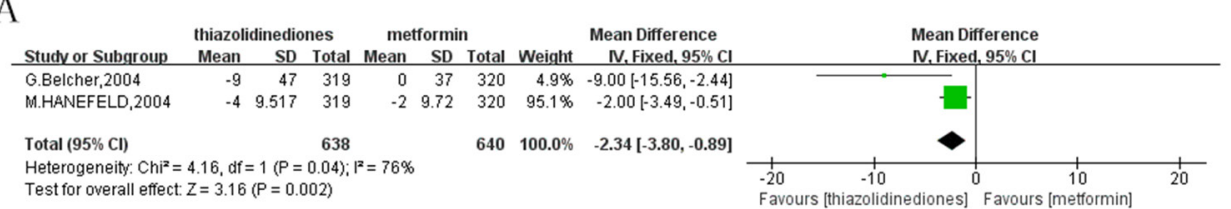

B

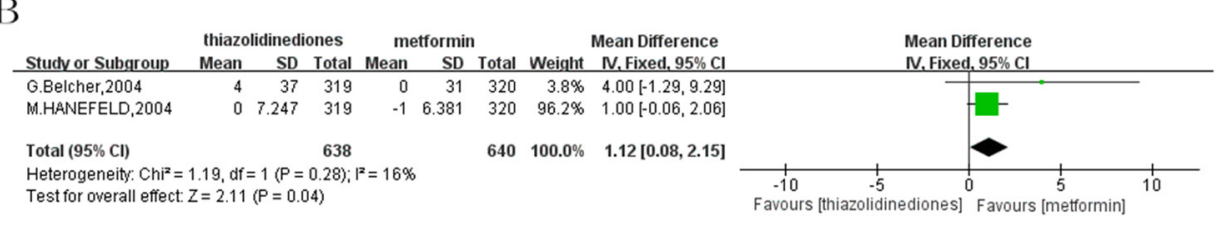

C

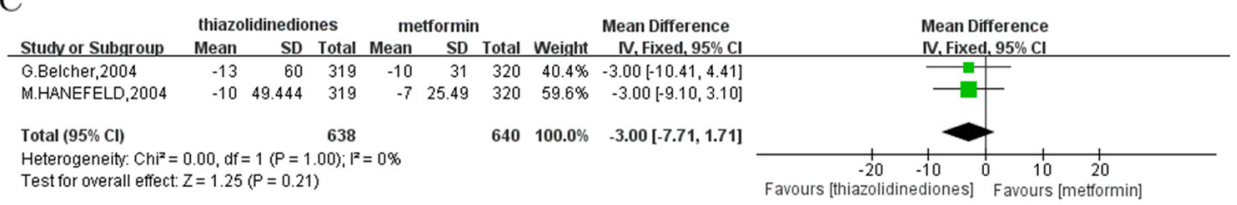

D

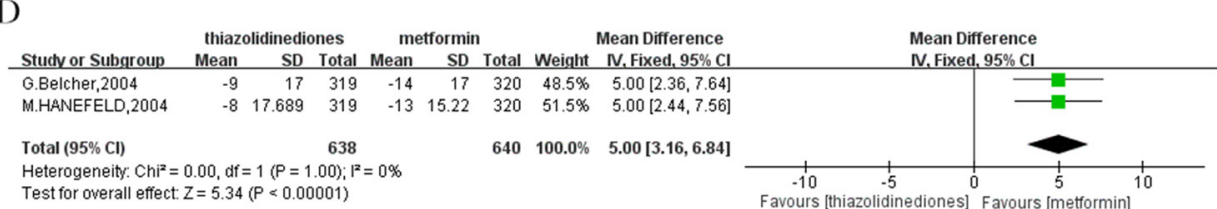

Figure 3: Forest plot of the improvement of liver enzymes from baseline to the end of treatment with adding-on to SUs. (A) ALT. (B) AST. (C) GGT. (D) AKP. ALT: alanine transaminase; AST: aspartate aminotransferase; GGT: gamma-glutamyl transpeptidase; AKP: alkaline phosphatase; SUs: sulfonylureas.

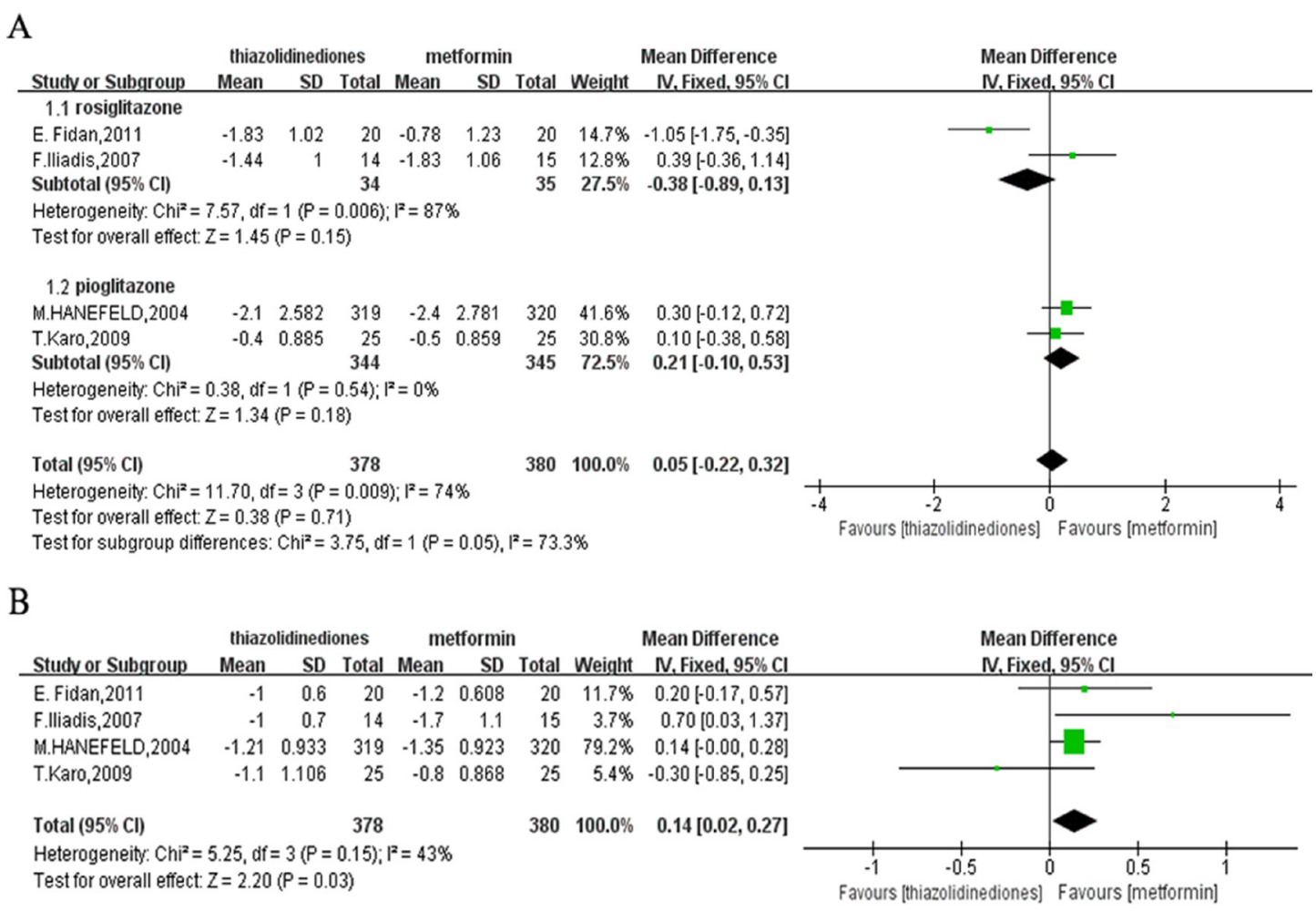

Figure 4: Forest plots comparing the effects of lowering glucose. (A) FPG. (B) HbA1C. FPG: fasting plasma glucose; HbA1C: glycosylated hemoglobin. 
AKP. Increased AKP mainly indicates the pathological changes in biliary flow, and highly concentrated bilirubin in serum causing damage to hepatocytes [38]. In contrast to metformin group, weaker improvement of AKP level for TZDs group may manifest less remission, which coincided with the finding of increased AKP levels for rosiglitazonetreated Akita animals after cholestasis liver injury [39]. Apart from the use of TZDs or metformin alone, the relevant results of combination with SUs resembled with the monotherapy. It revealed the significant decrease of ALT and similar capabilities in lowering GTT between
TZDs and metformin, and stronger effect on the reduction of AST and AKP when combining SUs with metformin. Evidence has emerged that the hepato-protective impact of gliclazide was more prominent in patients with chronic liver disease. And one possible explanation was due to the innate characteristics of gliclazide, which is known as a freeradical scavenger [40]. Thus it could be seen that SUs would play a supporting role in the effect on the liver function.

Due to differences in their in vivo metabolic pathways and affinity of binding with PPAR- $\gamma$, rosiglitazone and pioglitazone demonstrate shorter accumulation than

A

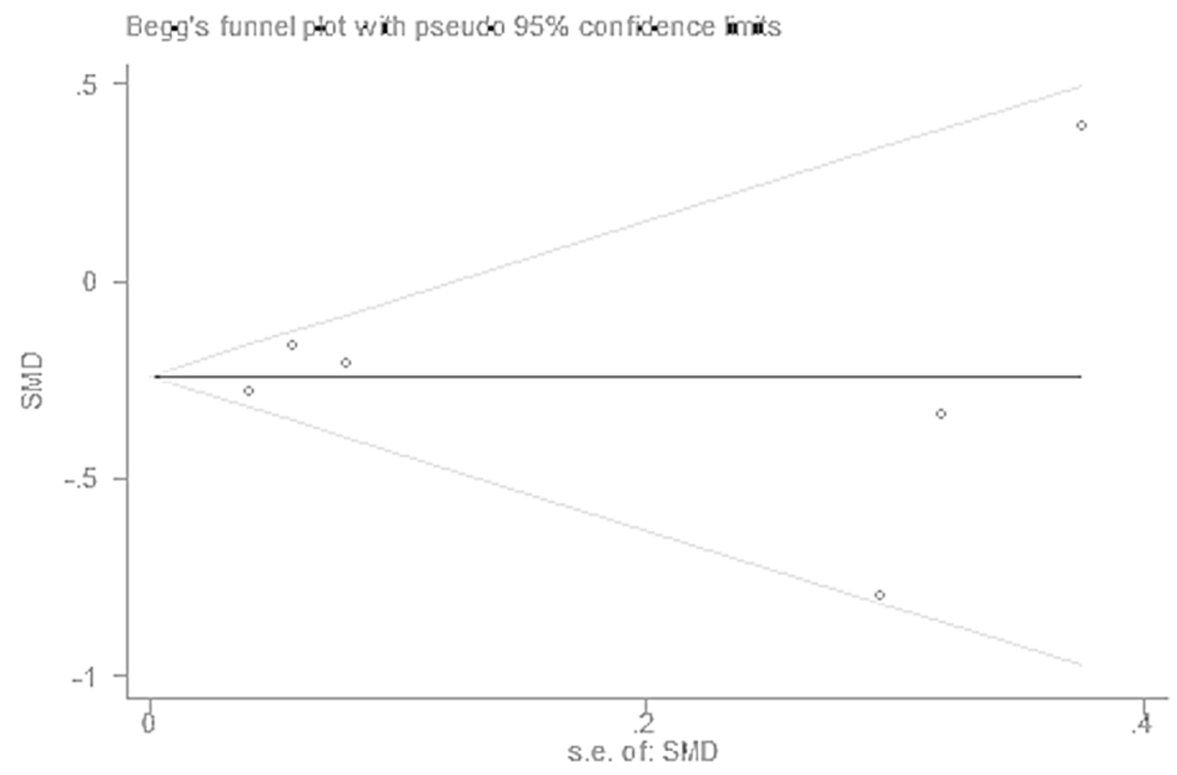

B

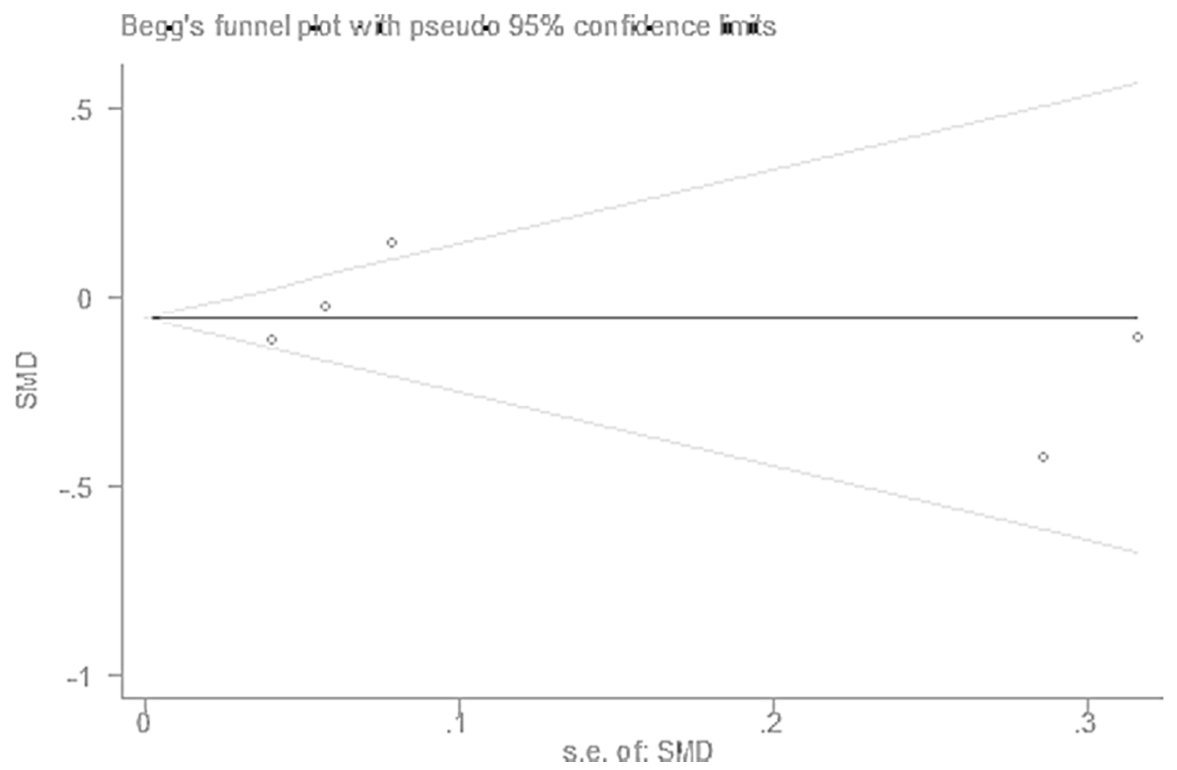

Figure 5: Funnel plots of ALT (A) and AST (B) revealed no significant publication bias. SMD: standardized mean difference; s.e. of SMD: standard error of standardized mean difference. 
troglitazone, thereby reducing their relative hepatotoxicity [19]. However, the limited number of randomized trials available does not allow us to draw any definitive conclusion about the different efficacy of the two TZDs on improvement of abnormal liver enzymes, especially with limited research choosing rosiglitazone as intervention.

In terms of glucose-lowering effect, our study revealed that metformin lowered $\mathrm{HbA1C}$ more significantly than TZDs, while both insulin-sensitizers showed the similar effect on reducing FPG. Greater reduction in $\mathrm{HbA} 1 \mathrm{C}$ indicated greater improvement in glycemic control. Metformin acting as the first-line hypoglycemic drug improves hepatic insulin resistance mainly by decreasing hepatic expression of TNF- $\alpha$, a cytokine that promotes insulin resistance $[25,27,41]$, ameliorates hepatic steatosis and decreases aminotransferase levels, thus causes reversal of fatty liver [27, 41]. Besides, beneficial action of metformin is thought to reduce excessive rates of hepatic glucose production. Moreover, glucose utilization by extra-hepatic tissues may also be enhanced through activation of AMPK [42]. However, TZDs enhance insulin sensitivity through stimulation of PPAR- $\gamma$, mainly acting in the skeletal muscles and liver as well as promoting adipogenesis of insulin-sensitive adipocytes [24]. Whereas, the only reduction by 0.27 at most of $\mathrm{HbA} 1 \mathrm{C}$ in the metformin group had little clinical significance. From the above, the efficacy on the reduction of plasma glucose may be equal between two insulin-sensitizers.

Nevertheless, our results should not be overinterpreted. We cannot ignore the fact that there was considerable heterogeneity among six trials in respect to sample size, type of experimental interventions, the duration of interventions, and the drug doses. Besides, there was a considerable discrepancy in the methodological quality across the included trials. Only one trial was regarded as being of high methodological quality [15]. Thus more researches on this field needs to be done further. And further prospective clinical studies are warranted to increase our understanding of the relationship between insulinsensitizers and improvement of abnormal liver enzymes.

\section{CONCLUSIONS}

In conclusion, the findings of this systematic review suggest that compared with metformin, TZDs may confer modest biological improvement of liver function in people with T2DM. More future researches need to focus on the specific factors related to the change of liver enzymes, such as improvement of hepatic insulin resistance. Moreover, we will take the morphologic features of liver into account in further studies.

\section{CONFLICTS OF INTEREST}

None.

\section{FUNDING}

This work was funded by National Natural Science Foundation of China Grants (No. 81670757, 81570742), Grant for the development of science and technology of Shandong Province (No. 2010GSF10228, 2012GGH11862, 2014GSF118118), Shandong Provincial Natural Science Foundation of China Grants (No. ZR2016HQ26, Y2008C73, ZR2010HM044), Shandong Provincial Science and Technology Development Program, China (No. 2009GGB14001), Grant for Excellent Young and Middle-aged Scientists of Shandong Province (No. 2004BS02016), and Grant for the development of science and technology of JiNan City (No. 201602172).

\section{REFERENCES}

1. Inzucchi SE, Bergenstal RM, Buse JB, Diamant $M$, Ferrannini E, Nauck M, Peters AL, Tsapas A, Wender R, Matthews DR, and American Diabetes Association (ADA), and European Association for the Study of Diabetes (EASD). Management of hyperglycemia in type 2 diabetes: a patientcentered approach: position statement of the American Diabetes Association (ADA) and the European Association for the Study of Diabetes (EASD). Diabetes Care. 2012; 35:1364-79.

2. Landman GW, de Bock GH, van Hateren KJ, van Dijk PR, Groenier KH, Gans RO, Houweling ST, Bilo HJ, Kleefstra N. Safety and efficacy of gliclazide as treatment for type 2 diabetes: a systematic review and meta-analysis of randomized trials. PLoS One. 2014; 9:e82880. https://doi. org/10.1371/journal.pone.0082880.

3. West J, Brousil J, Gazis A, Jackson L, Mansell P, Bennett A, Aithal GP. Elevated serum alanine transaminase in patients with type 1 or type 2 diabetes mellitus. QJM. 2006; 99:87176. https://doi.org/10.1093/qjmed/hcl116.

4. Asakawa M, Mitsui H, Akihisa M, Sekine T, Niitsu Y, Kobayashi A, Miyake A, Hashimoto N, Kawamura M, Ogawa Y. Efficacy and safety of sitagliptin for the treatment of diabetes mellitus complicated by chronic liver injury. Springerplus. 2015; 4:346. https://doi.org/10.1186/s40064015-1135-z.

5. Ratziu V, Goodman Z, Sanyal A. Current efforts and trends in the treatment of NASH. J Hepatol. 2015; 62:S65-75. https://doi.org/10.1016/j.jhep.2015.02.041.

6. Marchesini G, Petta S, Dalle Grave R. Diet, weight loss, and liver health in nonalcoholic fatty liver disease: Pathophysiology, evidence, and practice. Hepatology. 2016; 63:2032-43. https://doi.org/10.1002/hep.28392.

7. Vilar-Gomez E, Martinez-Perez Y, Calzadilla-Bertot L, Torres-Gonzalez A, Gra-Oramas B, Gonzalez-Fabian L, Friedman SL, Diago M, Romero-Gomez M. Weight loss through lifestyle modification significantly reduces features of nonalcoholic steatohepatitis. Gastroenterology. 
2015; 149:367-378. e5. https://doi.org/10.1053/j. gastro.2015.04.005.

8. Gouni-Berthold I, Papanas N, Maltezos E. The role of oral antidiabetic agents and incretin mimetics in type 2 diabetic patients with non-alcoholic fatty liver disease. Curr Pharm Des. 2014; 20:3705-15. https://doi.org/10.2174/138161281 13196660676.

9. Chan SP, Colagiuri S. Systematic review and meta-analysis of the efficacy and hypoglycemic safety of gliclazide versus other insulinotropic agents. Diabetes Res Clin Pract. 2015; 110:75-81. https://doi.org/10.1016/j.diabres.2015.07.002.

10. DerSimonian R, Laird N. Meta-analysis in clinical trials. Control Clin Trials. 1986; 7:177-88. https://doi. org/10.1016/0197-2456(86)90046-2.

11. Begg CB, Mazumdar M. Operating characteristics of a rank correlation test for publication bias. Biometrics. 1994; 50:1088-101. https://doi.org/10.2307/2533446.

12. Hanefeld M, Brunetti P, Schernthaner GH, Matthews DR, Charbonnel BH, and QUARTET Study Group. One-year glycemic control with a sulfonylurea plus pioglitazone versus a sulfonylurea plus metformin in patients with type 2 diabetes. Diabetes Care. 2004; 27:141-47. https://doi. org/10.2337/diacare.27.1.141.

13. Belcher G, Schernthaner G. Changes in liver tests during 1-year treatment of patients with Type 2 diabetes with pioglitazone, metformin or gliclazide. Diabet Med. 2005; 22:973-79. https:// doi.org/10.1111/j.1464-5491.2005.01595.x.

14. Iliadis F, Kadoglou NP, Hatzitolios A, Karamouzis M, Alevizos M, Karamitsos D. Metabolic effects of rosiglitazone and metformin in Greek patients with recently diagnosed type 2 diabetes. In Vivo. 2007; 21:1107-14.

15. Kato T, Sawai Y, Kanayama H, Taguchi H, Terabayashi T, Taki F, Yamada K, Yamazaki Y, Hayakawa N, Suzuki A, Oda N, Katada N, Itoh M. Comparative study of lowdose pioglitazone or metformin treatment in Japanese diabetic patients with metabolic syndrome. Exp Clin Endocrinol Diabetes. 2009; 117:593-99. https://doi. org/10.1055/s-0029-1202792.

16. Fidan E, Onder Ersoz H, Yilmaz M, Yilmaz H, Kocak M, Karahan C, Erem C. The effects of rosiglitazone and metformin on inflammation and endothelial dysfunction in patients with type 2 diabetes mellitus. Acta Diabetol. 2011; 48:297-302. https://doi.org/10.1007/s00592-011-0276-y.

17. Belcher G, Lambert C, Edwards G, Urquhart R, Matthews DR. Safety and tolerability of pioglitazone, metformin, and gliclazide in the treatment of type 2 diabetes. Diabetes Res Clin Pract. 2005; 70:53-62. https://doi.org/10.1016/j. diabres.2005.02.011.

18. Shon J, Abernethy DR. Application of systems pharmacology to explore mechanisms of hepatotoxicity. Clin Pharmacol Ther. 2014; 96:536-37. https://doi. org/10.1038/clpt.2014.167.

19. Hu D, Wu CQ, Li ZJ, Liu Y, Fan X, Wang QJ, Ding RG. Characterizing the mechanism of thiazolidinedione- induced hepatotoxicity: An in vitro model in mitochondria. Toxicol Appl Pharmacol. 2015; 284:134-41. https://doi. org/10.1016/j.taap.2015.02.018.

20. Shao N, Kuang HY, Hao M, Gao XY, Lin WJ, Zou W. Benefits of exenatide on obesity and non-alcoholic fatty liver disease with elevated liver enzymes in patients with type 2 diabetes. Diabetes Metab Res Rev. 2014; 30:521-29. https://doi.org/10.1002/dmrr.2561.

21. Abd El-Kader SM, El-Den Ashmawy EM. Non-alcoholic fatty liver disease: the diagnosis and management. World J Hepatol. 2015; 7:846-58. https://doi.org/10.4254/wjh. v7.i6.846.

22. Rizos CV, Kei A, Elisaf MS. The current role of thiazolidinediones in diabetes management. Arch Toxicol. 2016; 90:1861-81. https://doi.org/10.1007/s00204-0161737-4.

23. He L, Wondisford FE. Metformin action: concentrations matter. Cell Metab. 2015; 21:159-62. https://doi. org/10.1016/j.cmet.2015.01.003.

24. Kroker AJ, Bruning JB. Review of the structural and dynamic mechanisms of PPAR $\gamma$ partial agonism. PPAR Res. 2015; 2015:816856. https://doi.org/10.1155/2015/816856.

25. Scheen AJ. Pharmacokinetic and toxicological considerations for the treatment of diabetes in patients with liver disease. Expert Opin Drug Metab Toxicol. 2014; 10:839-57. https://doi.org/10.1517/17425255.2014.902444.

26. Mahady SE, Webster AC, Walker S, Sanyal A, George J. The role of thiazolidinediones in non-alcoholic steatohepatitis - a systematic review and meta analysis. J Hepatol. 2011; 55:1383-90. https://doi.org/10.1016/j.jhep.2011.03.016.

27. Omer Z, Cetinkalp S, Akyildiz M, Yilmaz F, Batur Y, Yilmaz C, Akarca U. Efficacy of insulin-sensitizing agents in nonalcoholic fatty liver disease. Eur J Gastroenterol Hepatol. 2010; 22:18-23. https://doi.org/10.1097/ MEG.0b013e32832e2baf.

28. Idilman R, Mizrak D, Corapcioglu D, Bektas M, Doganay B, Sayki M, Coban S, Erden E, Soykan I, Emral R, Uysal AR, Ozden A. Clinical trial: insulin-sensitizing agents may reduce consequences of insulin resistance in individuals with non-alcoholic steatohepatitis. Aliment Pharmacol Ther. 2008; 28:200-08.

29. Kim WR, Flamm SL, Di Bisceglie AM, Bodenheimer HC, and Public Policy Committee of the American Association for the Study of Liver Disease. Serum activity of alanine aminotransferase (ALT) as an indicator of health and disease. Hepatology. 2008; 47:1363-70. https://doi. org/10.1002/hep.22109.

30. Shigefuku R, Takahashi H, Nakano H, Watanabe T, Matsunaga K, Matsumoto N, Kato M, Morita R, Michikawa Y, Tamura T, Hiraishi T, Hattori N, Noguchi Y, et al. Correlations of hepatic hemodynamics, liver function, and fibrosis markers in nonalcoholic fatty liver disease: comparison with chronic hepatitis related to hepatitis C virus. Int J Mol Sci. 2016; 17:1545. https://doi.org/10.3390/ijms17091545. 
31. Lutchman G, Modi A, Kleiner DE, Promrat K, Heller T, Ghany M, Borg B, Loomba R, Liang TJ, Premkumar A, Hoofnagle JH. The effects of discontinuing pioglitazone in patients with nonalcoholic steatohepatitis. Hepatology. 2007; 46:424-29. https://doi.org/10.1002/hep.21661.

32. Harris EH. Elevated Liver Function Tests in Type 2 Diabetes. Clin Diabetes. 2005; 23:115-19.

33. Begriche K, Massart J, Robin MA, Bonnet F, Fromenty B. Mitochondrial adaptations and dysfunctions in nonalcoholic fatty liver disease. Hepatology. 2013; 58:1497-507. https:// doi.org/10.1002/hep.26226.

34. Browning JD, Horton JD. Molecular mediators of hepatic steatosis and liver injury. J Clin Invest. 2004; 114:147-52. https://doi.org/10.1172/JCI200422422.

35. Peta V, Torti C, Milic N, Focà A, Abenavoli L. Adiponectin serum level in chronic hepatitis $\mathrm{C}$ infection and therapeutic profile. World J Hepatol. 2015; 7:44-52. https://doi. org/10.4254/wjh.v7.i1.44.

36. Takeshita Y, Takamura T, Hamaguchi E, Shimizu A, Ota T, Sakurai M, Kaneko S. Tumor necrosis factor- $\alpha$ induced production of plasminogen activator inhibitor 1 and its regulation by pioglitazone and cerivastatin in a nonmalignant human hepatocyte cell line. Metabolism. 2006; 55:1464-72. https://doi.org/10.1016/j. metabol.2006.06.016.
37. Everett L, Galli A, Crabb D. The role of hepatic peroxisome proliferator-activated receptors (PPARs) in health and disease. Liver. 2000; 20:191-99. https://doi.org/10.1034/ j.1600-0676.2000.020003191.x.

38. Poupon R. Liver alkaline phosphatase: a missing link between choleresis and biliary inflammation. Hepatology. 2015; 61:2080-90. https://doi.org/10.1002/hep.27715.

39. Hemmeryckx B, Gaekens M, Gallacher DJ, Lu HR, Lijnen HR. Effect of rosiglitazone on liver structure and function in genetically diabetic Akita mice. Basic Clin Pharmacol Toxicol. 2013; 113:353-60.

40. Lee JY, Kim G, Lee YH, Lee BW, Cha BS, Nam CM, Kang ES. Comparison of hepatocellular carcinoma risk between patients treated with glimepiride and gliclazide. Diabetes Metab. 2017 Aug 1. [Epub Ahead of Print]. 2017S1262-3636(17)30471-8.

41. Lin HZ, Yang SQ, Chuckaree C, Kuhajda F, Ronnet G, Diehl AM. Metformin reverses fatty liver disease in obese, leptin-deficient mice. Nat Med. 2000; 6:998-1003. https:// doi.org/10.1038/79697.

42. Krentz AJ. Comparative safety of newer oral antidiabetic drugs. Expert Opin Drug Saf. 2006; 5:827-34. https://doi. org/10.1517/14740338.5.6.827. 